This report was prepared as an account of work sponsored by an agency of the United States Government. Neither the United States Government nor any agency thereof, nor any of their employees, makes any warranty, express or implied, or assumes any legal liability or responsibility for the accuracy, completeness, or usefulness of any information, apparatus, product, or process disclosed, or represents that its use would not infringe privately owned rights. Reference herein to any specific commercial product, process, or service by trade name, trademark, manufacturer, or otherwise does not necessarily constitute or imply its endorsement, recommendation, or favoring by the United States Government or any agency thereof. The views and opinions of authors expressed herein do not necessarily state or reflect those of the United States Government or any agency thereof.

\title{
LASER ULTRASONIC GENERATION AT THE SURFACE OF A LIQUID METAL
}

\author{
J. B. Walter and K. L. Telschow \\ Idaho National Engineering Laboratory \\ EG\&G Idaho, Inc. \\ Idaho Falls, ID 83415-2209 \\ R. J. Conant \\ Department of Mechanical Engineering \\ Montana State University \\ Bozeman, MT 59717
}

\section{INTRODUCTION}

The noncontacting nature of laser-based ultrasonic measurement has made it attractive in many applications. While most of the applications thus far involve solid samples, molten metals encountered in some stages of metal processing appear well suited to this technique. Possible applications for liquid metals include locating and characterizing the liquid/solid interface; characterizing a surface layer, contaminant, or alloying constituent; and measuring the temperature of the surface or of the bulk. The aim of this work was to characterize laser generation of ultrasound in liquid metals to facilitate the development of techniques for these applications.

This paper describes measurements of the angular distribution and energy dependence of laser-generated ultrasound in mercury at ambient temperature. Mercury was chosen as the model liquid metal because it is liquid at room temperature, and so should have negligible temperature gradients beyond those produced in the laser generation process, and because it exhibits a very shallow optical penetration depth. This paper also briefly describes the theory of laser generation by thermoelastic and ablation sources at the surface of a liquid metal. A simple, intuitive, phenomenological model for the partition of energy between thermoelastic and ablation source mechanisms is presented.

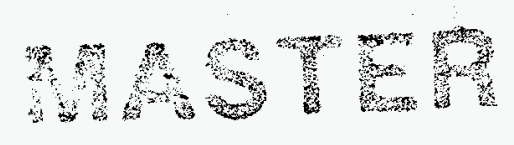




\section{DISCLAIMER}

Portions of this document may be illegible in electronic image products. Images are produced from the best available original document. 
The angular distribution of ultrasound was measured with the setup depicted in Figure 1. A horizontal glass cylinder, $50 \mathrm{~mm}$ in diameter, was half filled with mercury. ${ }^{a}$ Ultrasound was generated on the axis of the cylinder by $10 \mathrm{~ns}, 1.06 \mu \mathrm{m}$ pulses from a Nd-YAG laser, with the energy distributed over a spot approximately $2 \mathrm{~mm}$ in diameter. The ultrasonic waves were detected at the mercury/glass interface with a confocal Fabry-Perot detector at an adjustable angle $\theta$. The ultrasonic intensities measured for $16 \mathrm{~mJ}$ Nd-YAG pulses are tightly directed normal to the surface as evident in Figure 2.

There are two important modes of production of ultrasound for a pulsed laser incident on the surface of a liquid metal, thermoelastic expansion and ablation. The theory for thermoelastic expansion on a liquid metal surface was treated previously [1][2]. Heating at a point within the bulk of the liquid produces a monopole volume expansion, but the liquid free surface boundary condition results in an acoustic

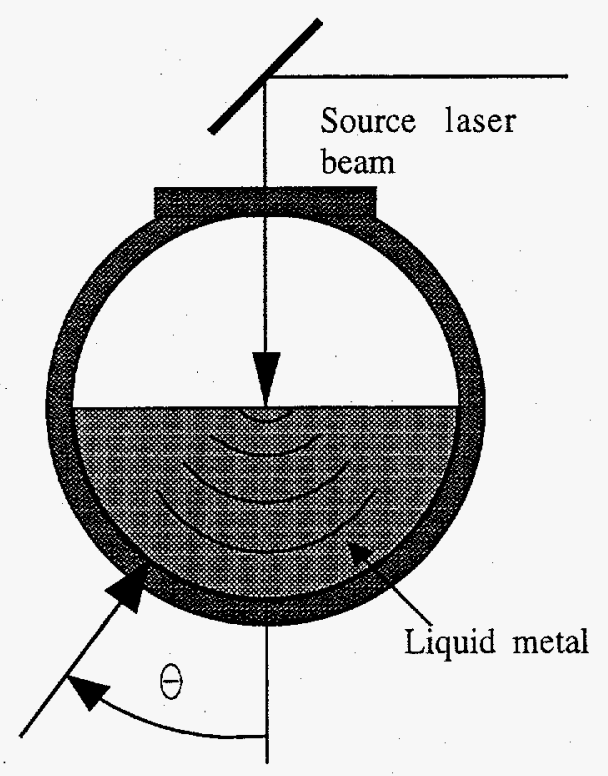

Detection laser beam

Fig. 1. Experimental arrangement for measuring the angular distribution of ultrasound generated by laser pulses on the surface of a pool of mercury.

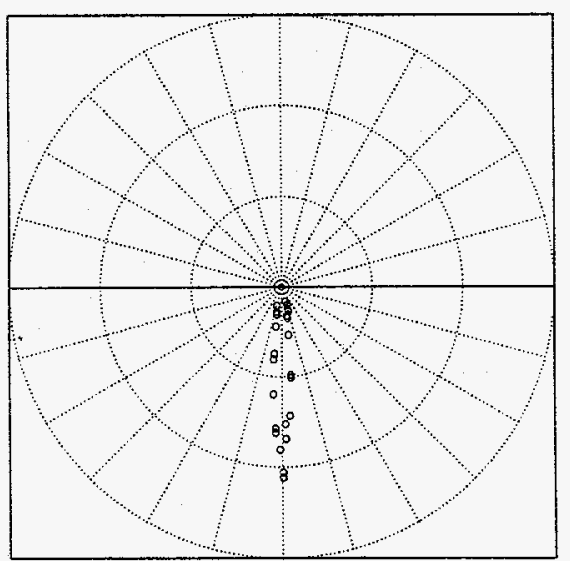

Fig. 2. Angular distribution of ultrasonic intensity for $16 \mathrm{~mJ}, 2 \mathrm{~mm}$ diameter, $1.06 \mu \mathrm{m}$ Nd-YAG laser pulse.

a The glass cylinder was sealed to avoid exposure to mercury vapor. 
dipole source as indicated in Figure 3. The radiated acoustic pressure amplitude in the liquid is given by

$$
p=i m p \omega \cos (\theta)(i k r-1) \frac{e^{i(k r-\omega t)}}{4 \pi r^{2}}
$$

where $\mathrm{m}$ is the dipole moment, $\rho$ is the liquid density, $\omega$ is the angular frequency, and $\mathrm{r}, \theta$ are the co-ordinates indicated in Figure 3. An ablative source acts as a point force, which also produces a dipole source with the same $r, \theta$ dependence of Equation (1) [1]. Both sources lead to a far-field intensity pattern proportional to

$$
\frac{\cos ^{2}(\theta)}{r^{2}}
$$

If the laser spot is Gaussian, with radius a, the far-field intensity is proportional to

$$
\frac{\cos ^{2}(\theta)}{r^{2}} e^{-\frac{(k a \sin (\theta))^{2}}{2}}
$$

where $\mathrm{k}$ is the wave number. The last factor, the Gaussian in ka $\sin \theta$, arises from diffraction of the finite beam distribution. The $\cos ^{2} \theta$ factor varies by only $8 \%$ for the angles displayed in Figure 2, hence the sharp directivity observed is due to the finite laser spot size. When replotted as ultrasonic amplitude as a function of angle, as in Figure 4, it is evident that Equation (3), plus a constant, can be fit to the data. Using $\mathrm{ka}=14$ from the width parameter of the Gaussian, and a nominal frequency of $4 \mathrm{MHz}$, we get $\mathrm{a}=0.8 \mathrm{~mm}$ for a spot diameter of $2.3 \mathrm{~mm}$, consistent with our estimate of $2 \mathrm{~mm}$.

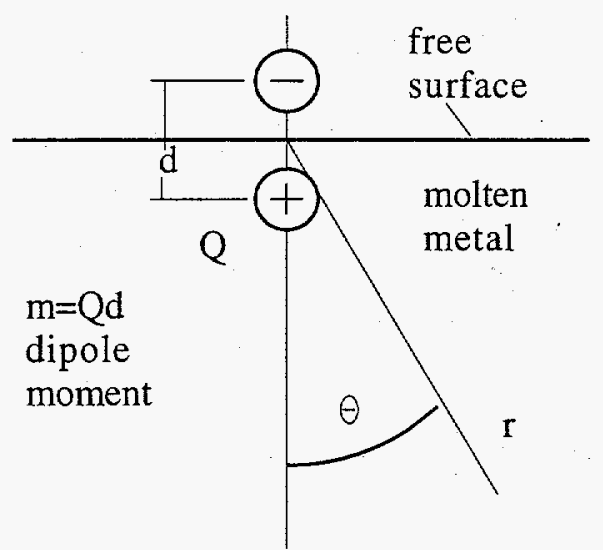

Fig. 3. Thermoelastic expansion from a point source on the free surface of a liquid metal.

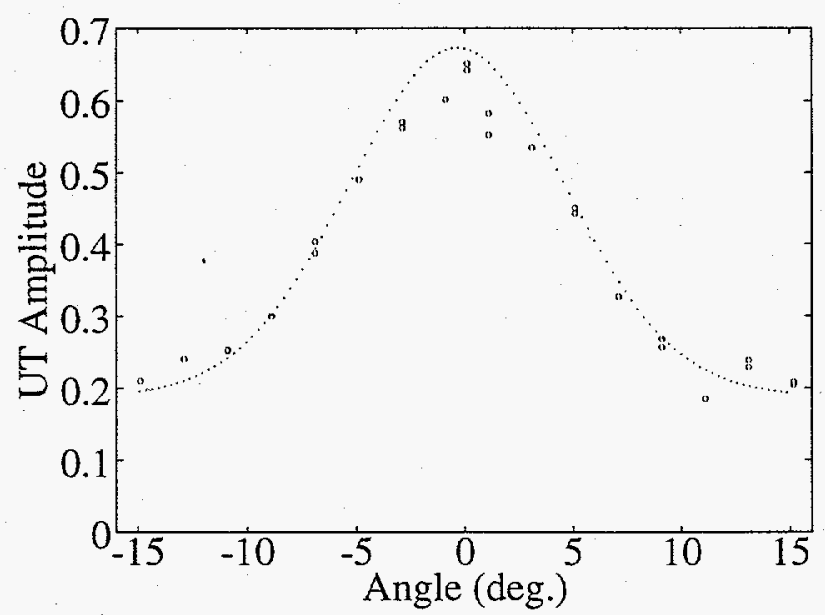

Fig. 4. Angular distribution of ultrasonic amplitude in Figure 2. The dotted curve is a constant plus Equation (3) fitted to the data. 


\section{ENERGY DEPENDENCE}

The dependence of the ultrasonic amplitude on energy in the Nd-YAG pulse was measured as shown in Figure 5. The ultrasonic wave was detected with a $10 \mathrm{MHz}$, $1 \mathrm{~mm}$ diameter, piezoelectric transducer. The mercury depth was $13 \mathrm{~mm}$, with the transducer $11 \mathrm{~mm}$ below the surface, so the ultrasonic amplitude measurements represent an average over $\Delta \theta<3^{\circ}$. The laser energy, adjusted by sliding thin glass plates in and out of the beam, was measured at each point. The spot size was measured photographically.

The ultrasonic amplitude is expected to vary linearly with laser pulse energy for thermoelastic generation, and also for ablative generation at low energies. For thermoelastic generation, the amplitude scales with the thermal expansion, and, therefore, with the absorbed pulse energy. For ablation, it will scale with the momentum of the ejected material, which is proportional to the mass ejected and the absorbed pulse energy above the ablation threshold.

Figure 6 shows the measured ultrasonic amplitudes for two spot sizes vs. incident laser pulse energy. The dashed lines show the expected slope for a linear dependence. Both data sets exhibit two linear regions, corresponding to thermoelastic expansion at lower energies and ablation at higher energies. A visible "spark" and an audible "snap" were observed for data points near the higher dashed line. For low laser energies, the absorbed incident light causes thermoelastic expansion. At high energies, ablation

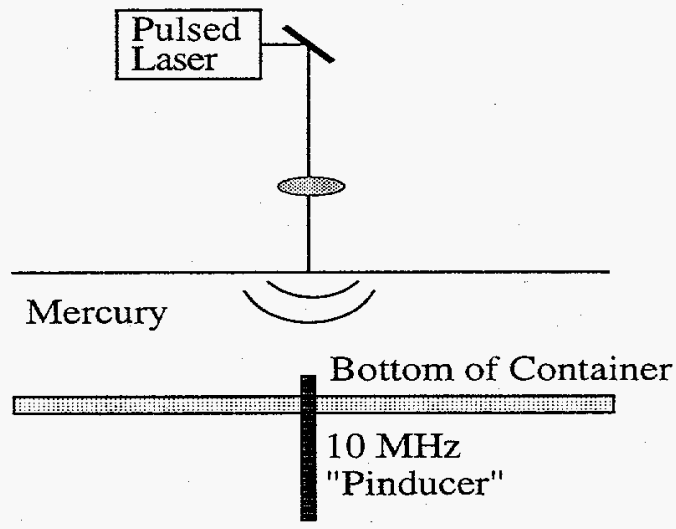

Fig. 5. Ultrasonic amplitude on epicenter was measured with a piezoelectric transducer.

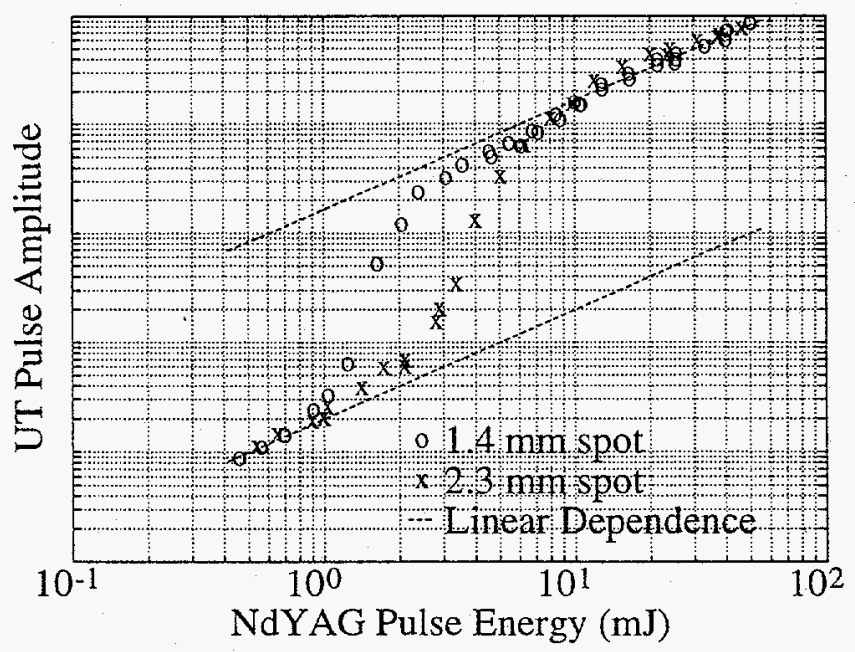

Fig. 6. The ultrasonic amplitude varies linearly with the Nd-YAG pulse energy in both the thermoelastic and ablation regimes. In the transition region depends on the spot size. 
causes material to be ejected. This material can absorb or scatter the incident light, resulting in a lower fraction of incident laser pulse energy actually being absorbed. Consequently, the ultrasonic amplitude is expected to saturate at sufficiently high laser pulse energies; however, no perceptible saturation was observed. The most striking feature of Figure 6 is the rapid increase of the ultrasonic amplitude with laser energy in the transition region between thermoelastic and ablation source mechanisms.

\section{SIMPLE THRESHOLD MODEL}

A simple threshold model is presented to explain Figure 6. The incident pulse energy, up to a threshold energy density, heats the liquid to the point of vaporization, producing ultrasound by thermoelastic expansion. Absorbed pulse energy beyond the threshold ejects material from the pool, producing ultrasound by ablation. From Figure 6 , it is evident that the ablation mechanism is two orders of magnitude stronger than the thermoelastic mechanism in mercury.

It is reasonable to assume that the amount of material ablated is proportional to the energy in the absorbed laser pulse that is above the threshold for ablation. Figure 7 shows the portion of the laser pulse, assumed a Gaussian, that contributes to ablation. The absorbed intensity is

$$
I=I_{0} e^{-\frac{r^{2}}{2 a^{2}}}
$$

where $\mathrm{I}_{0}$ is the peak energy density and a is the spot width parameter. Using cylindrical symmetry, the fraction of absorbed pulse energy contributing to ablation is

$$
F=\iint_{I>I_{t h}}\left(I-I_{t h}\right) r d r d \phi / \iint I r d r d \phi
$$

where $I_{\text {th }}$ is the threshold energy density, and the integral in the numerator is over the portion of the spot for which $I>I_{t h}$. For $I_{0}<I_{t h}, F=0$, while for $I_{0}>I_{t h}$

$$
F=1-\left(I_{t h} / I_{0}\right)\left[1+\ln \left(I_{0} / I_{t h}\right)\right]
$$

Therefore, the ultrasonic amplitude is predicted to have the form

$$
A \propto\left[(1-F)_{\text {therm }}+(\gamma F)_{a b l a t}\right] \pi a^{2} I_{0}
$$

where the subscripts therm and ablat are reminders of the source mechanisms and $\gamma$ is the strength of the ablation mechanism relative to thermoelastic expansion. Noting that

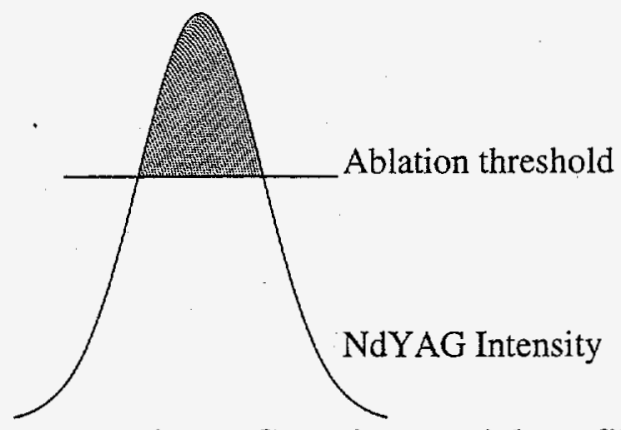

Fig. 7. Absorbed laser energy, assuming a Gaussian spatial profile. The absorbed energy density below threshold heats the liquid, with that above threshold, in the shaded region, causing ablation. 
the laser spot size enters into Equation (7) as a multiplicative factor, this model suggests plotting the data as ultrasonic pulse amplitude per unit area illuminated vs. the incident pulse energy, as shown in Figure 8. The data for both spot sizes fall on a single curve as predicted by the model.

There are two parameters in the model, $\mathrm{I}_{\mathrm{th}}$ and $\gamma$. Both depend on the thermal and optical properties of the liquid metal, with $\mathrm{I}_{\mathrm{th}}$ also dependent on the temperature of the liquid. Both are calculable in principle.

\section{CONCLUSIONS}

Both thermoelastic expansion and ablation cause similar ultrasound generation in liquid metals. Laser-generated ultrasonic pulses are directed perpendicular to the surface for liquid metals, for both mechanisms. The ultrasonic pulse amplitude is proportional to the absorbed laser pulse energy for both mechanisms. The simple threshold model presented for ablation describes the transition from thermoelastic generation. The model's two parameters are material dependent. For mercury, ablation produces about 100 times larger ultrasonic pulse amplitudes than thermoelastic expansion and has a threshold of about $6 \mathrm{MW} / \mathrm{cm}^{2}$. Molten metals offer a good medium for studying ablation, since the surface is always refreshed and ablation, at least for mercury, dominates ultrasonic generation above the threshold.

\section{ACKNOWLEDGMENTS}

The work described in this paper was supported by the Interior Department's Bureau of Mines under Contract No. J0134035 through DOE Idaho Operations Office Contract DE-AC07-76ID01570.

\section{REFERENCES}

1. S. Temkin, Elements of Acoustics, (John Wiley \& Sons, New York, NY, 1981), pp. 269-366.

2. F. V. Bunkin, A. A. Kolomensky and V. G. Mikalovich, Lasers in Acoustics, (Harwood Publishers, Philadelphia, PA, 1991)

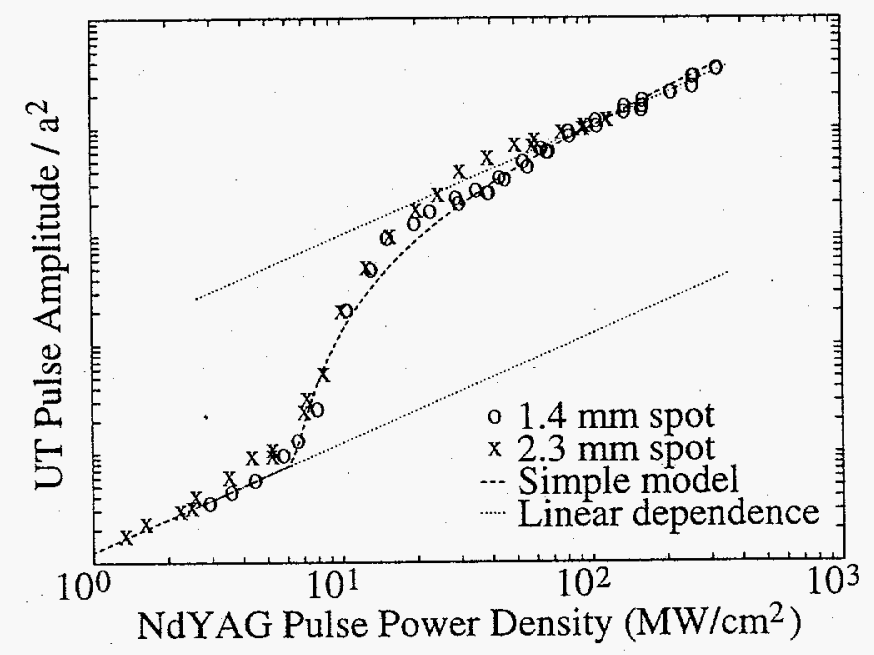

Fig. 8. The simple ablation model of Equations (6) and (7), shown as the dashed curve, displays the essential physics for the transition from thermoelastic expansion to ablation ultrasound production mechanisms. 